\title{
Negative pressure wound therapy promotes vessel destabilization and maturation at various stages of wound healing and thus influences wound prognosis
}

\author{
ZHANJUN MA, KANGQUAN SHOU, ZONGHUAN LI, CHAO JIAN, BAIWEN QI and AIXI YU \\ Department of Orthopedics, Zhongnan Hospital of Wuhan University, Wuhan, Hubei 430071, P.R. China
}

Received September 30, 2015; Accepted January 20, 2016

DOI: $10.3892 /$ etm.2016.3083

\begin{abstract}
Negative pressure wound therapy (NPWT) has been observed to accelerate the wound healing process in humans through promoting angiogenesis. However, the potential biological effect and relevant molecular mechanisms, including microvessel destabilization, regression and endothelial cell proliferation in the early stage (1-3 days), and the neovascular stabilization and maturation in the later stage (7-15 days), have yet to be fully elucidated. The current study aimed to research the potential effect of NPWT on angiogenesis and vessel maturation, and investigate relevant association between mature microvessels and wound prognosis, as well as the regulatory mechanisms in human wound healing. Patients in the present study $(\mathrm{n}=48)$ were treated with NPWT or a petrolatum gauze, and relevant growth factors and vessel changes were detected using various experimental methods. NPWT increased the expression levels of angiogenin-2 (Ang-2), and decreased the expression levels of Ang-1 and ratios of Ang-1/Ang-2 in the initial stages of wound healing. However, in the latter stages of wound healing, NPWT increased the expression levels of Ang-1 and ratios of Ang-1/Ang-2, as well as the phosphorylation level of tyrosine kinase receptor-2. Consequently, microvessel pericyte coverage was gradually elevated, and the basement membrane was gradually supplied with new blood at the later stage of wound healing. In conclusion, NPWT may preferentially stimulate microvessel destabilization and regression in the early stage of wound healing, and as a consequence, increase angiogenesis. Subsequently, in the later stage of wound healing, NPWT may preferentially promote microvessel stabilization, thereby promoting microvessel maturation in human wounds through the angiogenin/tyrosine kinase receptor-2 signaling pathway. The results of the present
\end{abstract}

Correspondence to: Dr Baiwen Qi and Professor Aixi Yu, Department of Orthopedics, Zhongnan Hospital of Wuhan University, 169 Donghu Road, Wuhan, Hubei 430071, P.R. China

E-mail:wb002162@whu.edu.cn

E-mail: yuaixi@whu.edu.cn

Key words: negative pressure wound therapy, pericyte, vessel maturation, wound prognosis study results demonstrated that NPWT was able to accelerate wound healing speed, and thus influence wound prognosis, as a result of an abundance of mature microvessels in human wounds.

\section{Introduction}

Negative pressure wound therapy (NPWT) has been extensively used for the treatment of acute and chronic wounds, and the underlying molecular mechanisms has been elucidated (1). It has recently been suggested that cutaneous wound healing is a processes involving elevated levels of neovascularization (2), and three different processes of neovascularization serve as a the minimum requirements for poor wound healing, including the following (3): Capillary regeneration (angiogenesis) and pericyte recruitment (vessel maturation), followed by conductance microvascular growth (arteriogenesis) (4). Recently, it has been revealed that microvascular maturation may be an important therapeutic target for neovascularization, as the lack of microvessel mature processes would weaken efficient perfusion of microvessels and prosperous growth of conductance microvessels (5-7).

Neovascularization is a multistage process involving the formation of arteries and veins, and is considered to have an important role in tissue repair and reconstruction. In the initial phases, angiogenesis is dependant on the degradation of the extracellular matrix and pre-existing blood vessels (8), followed by the formation of new endothelial tube composed of tip cells on the top of stalk cells (3). Subsequently, the cascaded growth factors and various cytokines that act on the vascular endothelial tubes are guided into the avascular area. As a result, the diameter of blood vessels increases and pericyte and vascular smooth cells are recruited to endothelial tubules and cover nascent endothelial cell tubules during arteriogenesis (3). Finally, the mature, functional microvascular circulatory system formed through neovascularization during the wound healing process, experiences enhanced vascular stability and blood flow perfusion is regulated (9). Mature capillaries provide increased blood flow perfusion, and provide an abundance of oxygen and nutrients to the wound tissue. Conversely, immature microvessels are typically inclined toward capillary destabilization and regression due to the absence of pericytes and, subsequently, blood flow perfusion is affected $(10,11)$. 
The positive therapeutic effects of NPWT have been generally accepted, including its ability to increase the quantity of angiogenesis and to increase the expression levels of growth factors and cytokines in the wound, thereby increasing blood flow perfusion and accelerating the wound healing process (12-15). However, whether the new blood vessels were mature, integrated and functional following NPWT, and how the relevant signal pathway regulates angiogenesis and vessel maturation, has yet to be reported conclusively in the literature. Furthermore, the process by which neovascularization was altered at various stages of the wound healing process, and the association between blood vessel maturity and wound prognosis, are only partially understood.

A recent study suggested that angiogenesis and vessel maturation were regulated primarily by the angiogenin (Ang) family (3). The family is comprised of 4 ligands, including Ang-1, Ang-2, Ang-3 (in mice) and Ang-4 (in humans), that bind the endothelial receptor tyrosine kinase, Tie-2 (11). It has previously been demonstrated that Tie- 2 is primarily expressed in endothelial cells in neovascularized sites and is associated with microvascular sprouting, branching, remodeling, maturation and stabilization (3). Ang-1 is primarily expressed in blood vessel mural cells (pericytes) $(16,17)$, and has an important role in regulating vessel maturation and endothelial cell migration, adhesion and survival (18). By contrast, Ang-2 acts as an antagonist and inhibits Ang-1 induced phosphorylation of Tie- 2 in the endothelium $(16,19,20)$. In addition, Ang-2 is able to disrupt the connection between cells in the endothelium and perivascular cells, promoting vascular regression and destabilization (18). Ang-3 is a Tie-2 receptor agonist, and is expressed in the endothelial cells of mice, whilst Ang- 4 served is also an agonist of the Tie-2 receptor, and is exclusively expressed in human lung tissue (21). Based upon the aforementioned findings, the present study primarily assessed the expression levels of Ang-1, Ang-2 and Tie-2.

In the current study, 48 clinical patients with soft tissue defects were recruited and treated with NPWT or a petrolatum gauze at various time-points, and the relevant detection methods were applied. The effect of NPWT on the process of angiogenesis and vessel maturation at different stages of wound healing was detected and, simultaneously, the associated signal pathway was explored. Finally, the association between wound prognosis and vessel maturation was assessed.

\section{Patients and methods}

Patients and grouping. Between January 2013 and January 2015,48 patients with soft tissue defects were recruited. Patients treated with NPWT $(n=26)$ served as the experimental group, and the remaining patients $(n=22)$ were treated with a petrolatum gauze and served as the control group. All patients were treated in the Department of Orthopedics, Zhongnan Hospital of Wuhan University (Wuhan, China). The present study was approved by the local ethical committee (approval no. 2012039; Zhongnan Hospital of Wuhan University) and written informed consent was obtained from all patients. The inclusion criteria was as follows: i) Patients experiencing acute soft tissue defects in the arms and legs, excluding those with any contraindication for NPWT; ii) the patients were between 18 and 50 years old; iii) patients were without active bleeding or malignancies; iv) patients did not have phlegmon; v) the etiology of all wounds was trauma; and vi) all wounds underwent debridement prior to the administration of NPWT or a petrolatum gauze.

In the present study, 23 men and 25 women with an age range of 30-50 years old were recruited, and demographics and the laboratory results of patient cohorts are displayed in Table I. Patients were assigned into the experimental or control group, according to patient's condition and inclusion criteria. Patients in the experimental group were further divided into day 1,3,7 or 15 subgroups and the wound beds were covered with a polyurethane foam dressing (Wuhan VSD Medical Science and Technology Corp., Hubei, China), with the pressure value constant set at continuous- $125 \mathrm{mmHg}$ to ensure that the vacuum-assisted closure device (Wego Biotechnology Co. Ltd., Weihai, China) did not affect activity or rest. The patients assigned to the control group were further divided into day $1,3,7$ or 15 subgroups and administered with a petrolatum gauze dressing. An antibiotic (2g cefpiramide; b.i.d; i.v.; Changlong Biochemical Pharmaceutical Co., Ltd., Huinan, China) was administered according to the results of a drug susceptibility test until infection had been completely controlled. The polyurethane foam dressing was replaced 2 times/week, and the gauze dressing was replaced as necessary.

Wound blood flow and perfusion detection. Wound surface blood flow perfusion was detected on days $1,3,7$, and 15 by a Laser Doppler Blood Perfusion Imager and a PeriScan PIM 3 system Perimed Ltd. (Stockholm, Sweden). The surface blood flow perfusion of each wound was assessed non-invasively without disturbing the wound at a distance of $15 \mathrm{~cm}$ and lasted for $6 \mathrm{~min}$. Concurrently, fresh granulation tissue from the center of the wound and margin were aseptically harvested by punching biopsy (Hengchang Steel Co., Ltd., Tangshan, China) on days 1, 3, 7 and 15 . Samples were subdivided into two, with one section fixed in $4 \%$ neutral paraformaldehyde (Aspen Bio, Wuhan, China) for histopathological and immunofluorescent investigations and the second section was stored in liquid nitrogen at $-80^{\circ} \mathrm{C}$ for protein analysis.

A local transposition flap or the use of a full-thickness skin graft was utilized to provide full coverage of the wound in the event that a red granulating wound bed was confirmed following NPWT or petrolatum gauze treatment $(22,23)$. The selection of methods for the secondary wound coverage was determined based on the growth of granulation tissue and exposure of tendon and bone. Wounds with adequate growth of granulation tissue were covered using the split or full thickness skin grafts; whereas wounds with exposed bone or tendons were covered using the transposition flap.

Immunohistochemical analysis. Samples were fixed in $4 \%$ neutral paraformaldehyde and embedded in paraffin. Serial cuts were made into 5- $\mu \mathrm{m}$ slices, sections underwent deparaffinized and were rehydrated, and the slices were then placed on to glass slides and stained with hematoxylin and eosin (Boster Biological Technology, Wuhan, China).

For immunohistochemical staining, antibodies against goat polyclonal Ang-1 (1:200; sc-6319), rabbit polyclonal Ang-2 (1:150; sc-20718) and Tie-2 (1:200; sc-9026), and mouse 
Table I. Patient demographics and laboratory results for the two patient cohorts.

\begin{tabular}{lccc}
\hline Parameter & Experimental group & Control group & P-value \\
\hline Patients, $\mathrm{n}$ & 26 & 22 & - \\
Age, years & $39.09 \pm 6.45$ & $40.27 \pm 6.00$ & 0.516 \\
Gender, F:M & $15: 11$ & $10: 12$ & 0.752 \\
Initial defect size, $\mathrm{cm}^{2}$ & $73.28 \pm 36.28$ & $77.42 \pm 36.16$ & 0.695 \\
Exposure of tendon and bone, case & $20(76.92)$ & $17(77.27)$ & 0.978 \\
Post-operative infection, case & $7(26.92)$ & $15(57.69)$ & 0.004 \\
Skin grafting, case & $21(80.77)$ & $6(27.27)$ & 0.000 \\
Transposition flap, case & $5(19.23)$ & $16(72.73)$ & 0.000 \\
Duration of hospital stay, days & $32.73 \pm 5.64$ & $43.32 \pm 12.16$ & 0.008 \\
\hline
\end{tabular}

Continuous variables are presented as mean \pm standard deviation and categorical variables are presented as n (\%). F, female; M, male.

monoclonal collagen type IV (1:200; sc-59814) and $\alpha$-smooth muscle actin ( $\alpha$-SMA; 1:200; sc-130616) were purchased from Santa Cruz Biotechnology, Inc. (Dallas, TX, USA) and served as primary antibodies in the present study. Initially, endogenous peroxidase was quenched with a $3 \%$ hydrogen dioxide; following this, citrate buffer was used for antigen retrieval, sections underwent microwave treatment (Galanz Group Ltd., Foshan, China) at $500 \mathrm{~W}$ for $5 \mathrm{~min}$. Following this, sections were incubated with the appropriate primary antibody at $4^{\circ} \mathrm{C}$ overnight. Subsequently, sections were washed three times with phosphate-buffered saline (PBS; Bioyear, Wuhan, China), and incubated with horseradish peroxidase-conjugated (HRP) goat anti-mouse (31430), goat anti-rabbit (31466) and rabbit anti-goat (31402) $\operatorname{IgG}(\mathrm{H}+\mathrm{L})$ secondary antibodies (all 1:500; all Invitrogen; Thermo Fisher Scientific, Inc., Waltham, MA, USA) for $30 \mathrm{~min}$. Sections were then incubated with an avidin-biotin complex (VECTASTAIN Elite ABC kit; Vector Laboratories, Inc. Burlingame, CA, USA) for $30 \mathrm{~min}$. Subsequently, the reaction was visualized with 3'3-diaminobenzidine (Dako, Glostrup, Denmark), and nuclei were stained using hematoxylin and eosin. Finally, all images were captured on a fluorescence microscope (BX51WI; Olympus Corporation, Tokyo, Japan).

Immunofluorescence analysis. To further observe the proliferation of microvascular endothelial cells and pericyte coverage, a double-labeling immunofluorescence technique was applied. Mouse monoclonal anti-CD31 (1:200; ab9498; Abcam, Cambridge, UK) and rabbit polyclonal anti-Ki67 (1:600; ab15580; Abcam) antibody markers were used to observe the proliferation of microvascular endothelial cells. Similarly, the pericyte coverage of microvessels was assessed using anti-CD31 and $\alpha$-SMA (1:400; ab124964; Abcam) antibodies. Sections were blocked with bovine serum albumin (Roche Diagnostics, Beijing, China) for $2 \mathrm{~h}$, and incubated with the required primary antibody for at $4^{\circ} \mathrm{C}$ overnight. Sections were washed three times with PBS then incubated with fluorescein isothiocyanate-conjugated goat anti-rabbit (65-6111) and Cyanine 3-conjugated goat anti-mouse (M30010) IgG (H+L) secondary antibodies (both 1:400; both Invitrogen) for $1 \mathrm{~h}$ in a dark environment. Following this, sections were incubated in 4',6-diamidino-2-phenylindole (Aspen Bio) to stain and visualize the nuclei. Images were captured using an Eclipse TE2000-E fluorescence microscope (Nikon Corporation, Tokyo, Japan), analyzed using Image-Pro Plus software (version 6.0; Media Cybernetics, Inc., Rockville, MD, USA).

Quantitation of the proliferating capillary index (PCI). The PCI was used to evaluate the proliferation of microvascular endothelial cells, and the ratio was expressed as proliferating microvascular endothelial cells (as determined by Ki67) divided by total number of microvessels (determined by CD31; Ki67/CD31). PCI was quantified by three independent investigators screening vessel hot spots with the highest microvessel density under a magnification field of x200.

Quantification of microvessel density (MVD). MVD quantification methods were used to evaluate the number of blood vessels number in the present study $(11,24)$. CD31 served as an endothelial cell marker, regardless of the presence or absence of lumen, and 3 separate sections of each sample were counted in 5 randomly selected areas with magnification x200. MVD was quantified and the average number of microvessels in each viewing field was recorded.

Quantification of the microvessel pericyte coverage index (MPI). MPI was applied to evaluated microvascular maturity, and values are expressed as $\alpha$-SMA divided by the number of microvessels observed in CD31 staining ( $\alpha$-SMA/CD31). A previously described method for quantification was applied to the present study $(11,25-28)$. A single endothelial cell was deemed as 1 unit of quantify, regardless of whether a tube was formed. A single pericyte was defined as a single layer of $\alpha$-SMA-positive cells colocalized with CD31-positive cells. MPI was quantified in a minimum of five non-overlapping microscopic areas per section, and sections were analyzed by three independent investigators under double-blind conditions. Pericyte coverage was expressed as the $\alpha-S M A / C D 31$ ratio.

Reverse transcription-quantitative polymerase chain reaction $(R T-q P C R)$. RT-qPCR was performed to quantitatively analyze mRNA expression levels. RNA $(\leq 30 \mathrm{mg}$, depending 
Table II. Primer sequences for reverse transcription-quantitative polymerase chain reaction.

\begin{tabular}{lll}
\hline Factor & \multicolumn{1}{c}{ Forward primer } & \multicolumn{1}{c}{ Reverse primer } \\
\hline Ang-1 & 5'-CTACCACCAACAACAGTGTCCTTC-3' & 5'-TTCTCTTCCTCTCTTTTTCCTCCC-3' \\
Ang-2 & 5'-GCGTTGATTTTCAGAGGACTTG-3' & 5'-GATGCTGCTTATTTTGCCGG-3' \\
$\alpha$-SMA & 5'-CTTGAGAAGAGTTACGAGTTGC-3' & 5'-GATGCTGTTGTAGGTGGTTTC-3' \\
Collagen IV & 5'-GGTGTTACAGGATTGGTGGGT-3' & 5'-GAAGGACACTGTGGGTCATCTATT-3' \\
GAPDH & 5'-GGTCGGAGTCAACGGATTTG-3' & 5'-GGAAGATGGTGATGGGATTTC-3' \\
\hline
\end{tabular}

Ang, angiogenin; $\alpha$-SMA; $\alpha$-smooth muscle actin.

on the tissue type) was disrupted in Buffer RLT (Qiagen AB, Sollentuna, Sweden) and homogenized. Total RNA was extracted using a RNeasy Mini kit (Qiagen AB), and $5 \mu \mathrm{g}$ RNA was reverse transcribed into cDNA using the RevertAid First Strand cDNA Synthesis kit (Fermentas; Thermo Fisher Scientific, Inc.) to a final reaction volume of $20 \mu \mathrm{l}$ and an S1000 Thermal Cycler (Bio-Rad Laboratories, Inc., Hercules, CA, USA) at $65^{\circ} \mathrm{C}$ for $5 \mathrm{~min}$, cooling on ice and $42^{\circ} \mathrm{C}$ for $60 \mathrm{~min}$, according to the manufacturer's protocol. The reaction was terminated by heating at $70^{\circ} \mathrm{C}$ for 5 min. Primer sequences are displayed in Table II.

Following DNase treatment to remove genomic DNA, RT-qPCR was performed to a final volume of $20 \mu \mathrm{l}$ using $1 \mu$ l template cDNA, $10 \mu \mathrm{l}$ SYBR qPCR mix (2X; Toyobo Co., Ltd., Osaka, Japan), $6.6 \mu 1$ diethylpyrocarbonate-treated water, $1 \mu \mathrm{l}$ forward primer $(5 \mu \mathrm{m}), 1 \mu \mathrm{l}$ reverse primer $(5 \mu \mathrm{m})$, $0.4 \mu \mathrm{l}$ ROX reference dye $(50 \mathrm{X})$ on a iQ5 Real-Time PCR Detection System (Bio-Rad Laboratories, Inc.). Respective negative (no cDNA) and RT controls were used for each gene. The thermocycling profile for SYBR Green RT-qPCR was as follows: Initially set at $95^{\circ} \mathrm{C}$ and sustained for $1 \mathrm{~min}$ for the initial denaturation step; followed by 40 cycles of degeneration with the temperature set at $95^{\circ} \mathrm{C}$, which was held for $15 \mathrm{sec}$, then set at $60^{\circ} \mathrm{C}$ and sustained for $15 \mathrm{sec}$ for annealing; and, finally, the elongation step, involving a $60 \mathrm{sec}$ hold at $72^{\circ} \mathrm{C}$. Each sample was run in triplicate and quantified using the $2^{-\Delta \Delta \mathrm{Cq}}$ method (29) to determine relative mRNA expression levels.

Western blot analysis. Samples were homogenized and total proteins were extracted using radioimmunoprecipitation assay buffer (Beyotime Institute of Biotechnology, Haimen, China). The concentrations of proteins were determined using a bicinchoninic acid assay kit (Beyotime Institute of Biotechnology). Proteins $(40 \mu \mathrm{g})$ were loaded onto a sodium dodecyl sulfate polyacrylamide gels (10\%; Aspen Bio) and run at $120 \mathrm{~V}$ for $90 \mathrm{~min}$. Proteins were then transferred to nitrocellulose membranes (Pall Life Sciences, Port Washington, NY, USA), and incubated $2 \mathrm{~h}$ with non-fat dry milk (5\%) in TBS-T (10 mM, Tris base pH 7.5; $150 \mathrm{mM}$ $\mathrm{NaCl}, 0.1 \%$ Tween-20; Aspen Bio) at room temperature. The membranes were incubated at $4{ }^{\circ} \mathrm{C}$ overnight with primary antibodies against Ang-1 (1:1,000; sc-6319), Ang-2 (1:1,000; sc-20718), p-Tie-2 (1:500; sc-130607), $\alpha$-SMA (1:2,000; sc-130616), GAPDH (1:5,000; sc-25778) and collagen type IV (1:1,500; sc-59814; all Santa Cruz Biotechnology, Inc.).
Subsequently, membranes were washed three times with TBS-T for $10 \mathrm{~min}$. Finally, membranes were incubated with the required HRP-conjugated goat anti-mouse (31430), goat anti-rabbit (31466) and rabbit anti-goat (31402) IgG $(\mathrm{H}+\mathrm{L})$ secondary antibodies for $1 \mathrm{~h}$ at room temperature, and an enhanced chemiluminescence substrate (Beyotime Institute of Biotechnology) was used to detect the membranes.

Statistical analysis. Data are presented as mean \pm standard deviation. Comparisons between the blood flow perfusion, proliferating capillary index, microvascular density, number of pericytes, microvessel pericyte coverage index, mRNA and protein expression level changes between control and NPWT groups at the same time point were conducted using Student's t-test. Differences between the groups at different time points were compared using one-way analysis of variance. All statistical analyses were performed using SPSS software (version 19; IBM SPSS, Armonk, NY, USA). P<0.05 was considered to indicate a statistically significant difference.

\section{Results}

Demographics and laboratory results of patient cohorts. There were no statistical differences in the age, gender, pre-operative initial defect size or exposure of tendon and bone ( $\mathrm{P}>0.05$; Table I). However, following the surgery the cultures of wound swabs revealed that the infection rate in the experimental group were significantly lower compared with the control group $(\mathrm{P}<0.05)$. All wounds were covered with skin grafting or a transposition flap, and results indicated that the cases of skin grafting in the experimental group were significantly higher $(\mathrm{P}<0.01)$ compared with those of the control group, while fewer cases were treated with transposition flap compared with the control group $(\mathrm{P}<0.01)$, and the hospital stay in experiment group was significantly shorter compared with the control group $(\mathrm{P}<0.05)$.

Blood flow perfusion. Blood flow perfusion results indicated that there was an abundance of areas of hyperperfusion and reperfusion following NPWT on days 7 and 15. However, in the control group, the hyperperfusion and reperfusion areas were decreased during this time point (Fig. 1A). The blood flow perfusion values gradually increased between day 3 and day 15 in the experimental group, in particular on days 7 and 15 , where the difference was statistically significant compared with the control group $(\mathrm{P}<0.05$; Fig. 1B). 
A

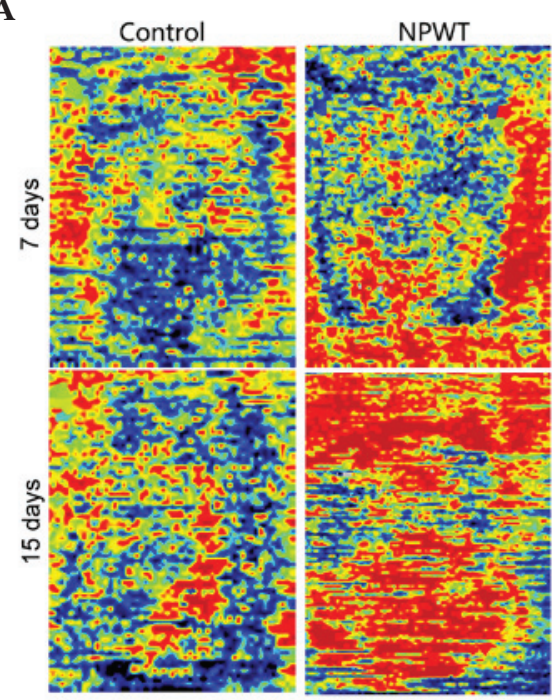

B

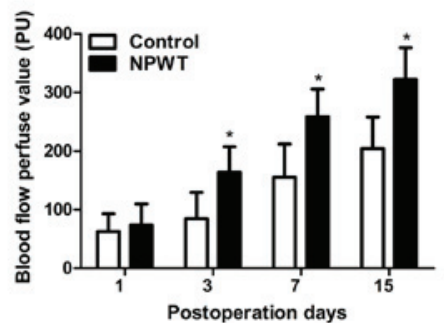

C

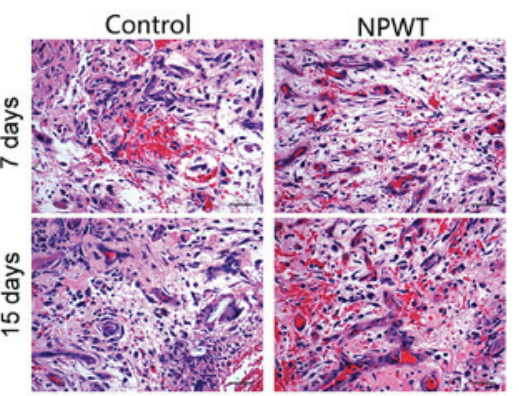

D

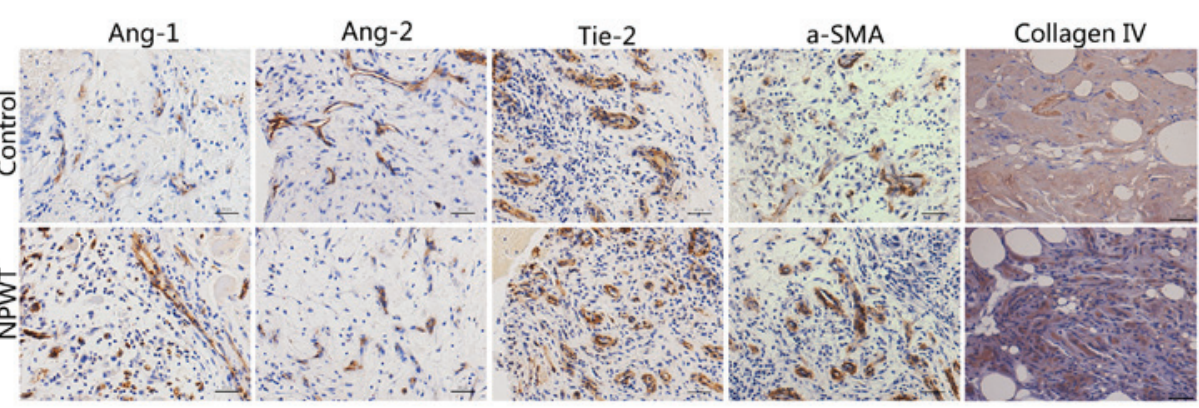

Figure 1. Microvessel blood flow perfusion changes and macroscopic wound healing in control and NPWT groups at different time points. (A) Microvessel blood flow perfusion was assessed on days 7 and 15 following surgery. (B) Statistical analysis of blood flow perfusion values in the two groups during wound healing. "P<0.05 vs. control. (C) Histological changes in the tow groups on day 15 (scale bar, $50 \mu \mathrm{m}$ ). (D) Immunohistochemical changes of the groups on day 7 (scale bar, $50 \mu \mathrm{m}$ ). Ang, angiogenin; $\alpha$-SMA, $\alpha$-smooth muscle actin; NPWT, negative pressure wound therapy.

A

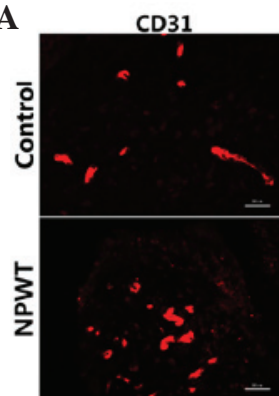

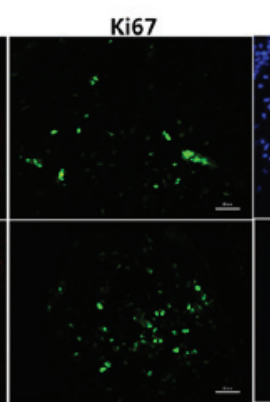

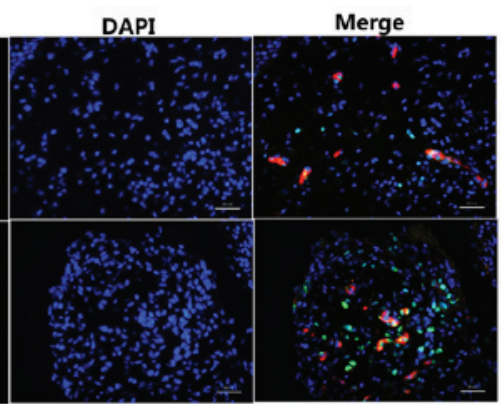

B

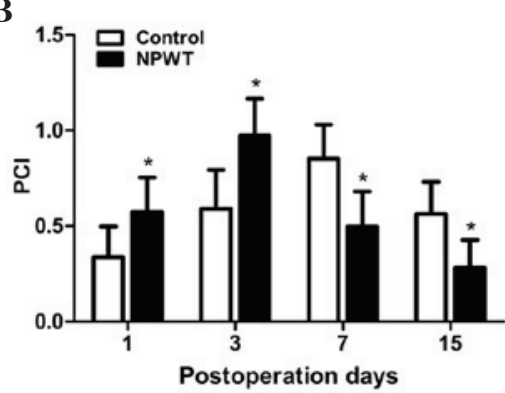

Figure 2. Immunofluorescence assay of proliferating endothelial cells in the wound in different groups. (A) Representative examples of double staining of Ki67 and CD31 (red, CD31; green, Ki67; nuclei, blue) in wound sections from the experimental and control groups on day 3 following surgery (magnification, x200). (B) Quantitative comparison of the PCI for the two groups. The PCI was used to evaluate the percentage of proliferating endothelial cells. Results are expressed as the mean \pm standard deviation. "P<0.05 vs. control group. DAPI, 4',6-diamidino-2-phenylindole; PCI, proliferating capillary index; NPWT, negative pressure wound therapy.

Histopathological assessments. Epidermal necrolysis and inflammatory cell infiltration was detected in the control group in the early stage of wound healing. In the later stage, there was an abundance of neovascularization accompanied with compactly and regularly arranged collagen fibers in the wounds of the experimental group. By contrast, a smaller number of nascent blood vessels were present in wounds in the control group, and collagen fibers had a disorganized distribution (Fig. 1C).
Immunohistochemical detection. Immunohistochemical staining revealed that Ang-1 was primarily expressed in pericytes, and in the experimental group, the positive staining of Ang-1 was markedly higher compared with the control group on the day 7 (Fig. 1D). By contrast, Ang-2 was predominately expressed in endothelial cells, and the positive staining of Ang-2 in experimental group was markedly lower compared with the control group on day 7. Furthermore, Tie-2 was primarily 
A

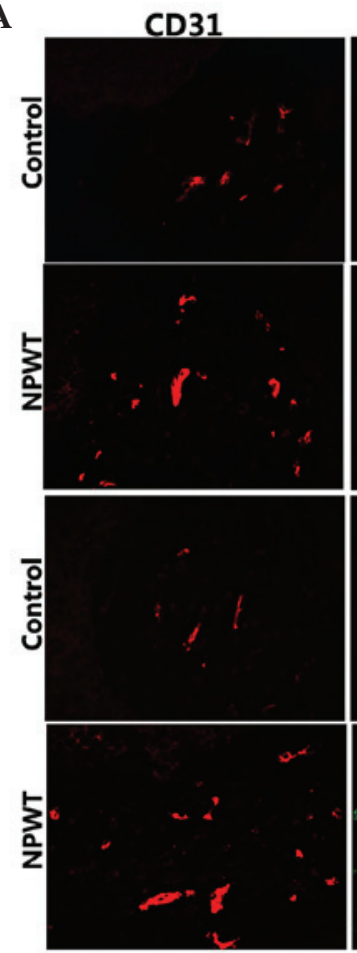

B

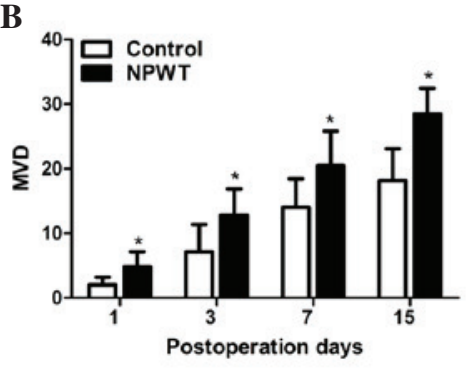

$\alpha$-SMA

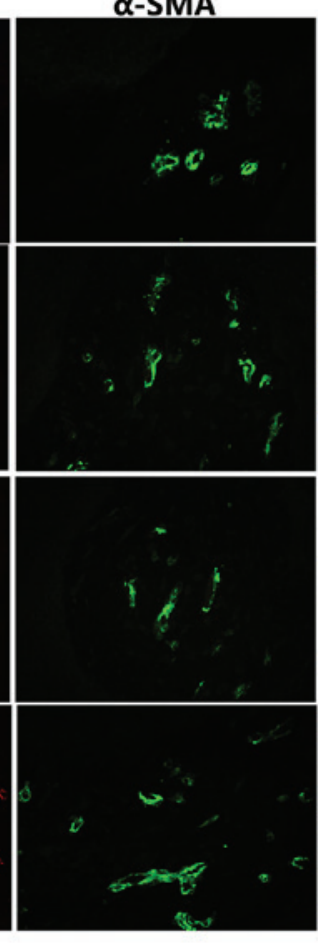

C

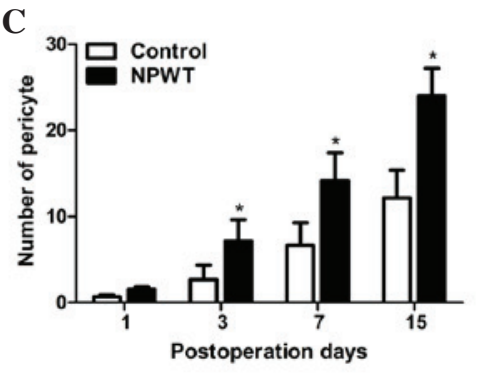

Merge

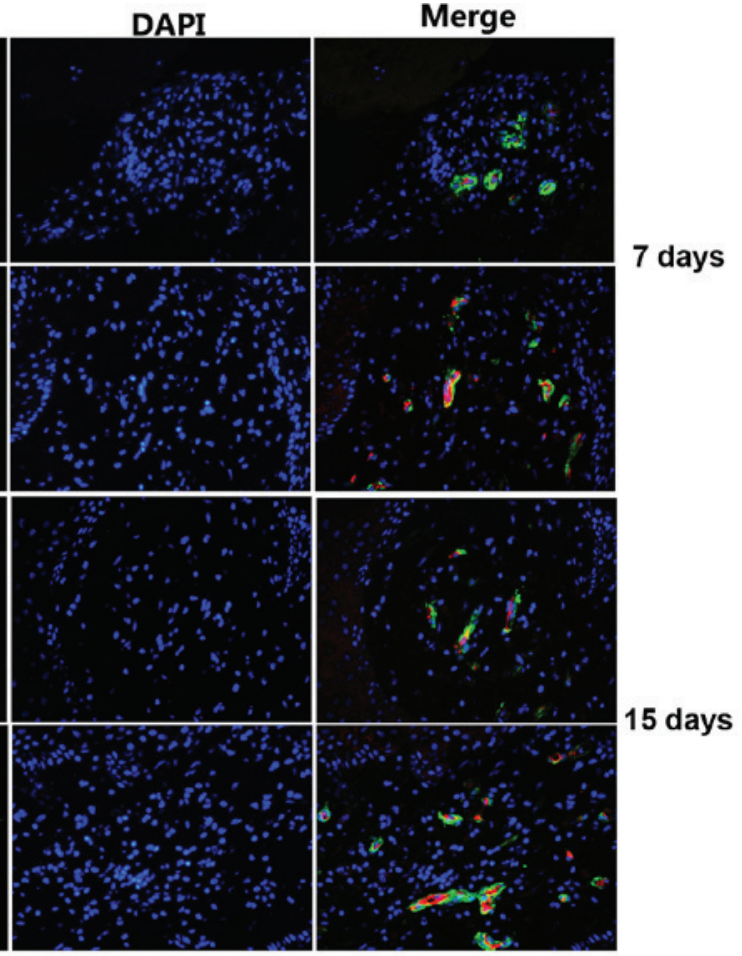

D

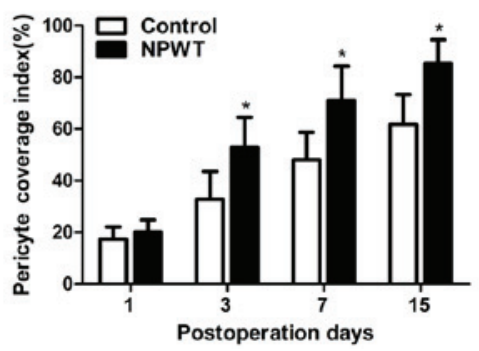

Figure 3. Immunofluorescence analysis of MVD and microvessel pericyte coverage in the two groups in the wound. (A) Samples from the groups were double stained to identify endothelial cells by CD31 (red) and pericyte by $\alpha$-SMA expression (green) (magnification, x200). (B) The MVD was quantified at different time points. (C) Quantitative comparison of the numbers of microvessel pericyte in the different groups and at different time points. (D) The microvessel pericyte coverage index was quantified and analyzed in the two groups. All statistical analysis results are expressed as the mean \pm standard deviation $(* \mathrm{P}<0.05)$. DAPI, 4',6-diamidino-2-phenylindole; $\alpha$-SMA, $\alpha$-smooth muscle actin; MVD, microvessel density; NPWT, negative pressure wound therapy.

present in endothelial cell and results revealed that on day 7 , the Tie-2-positive stained area was greater in the experimental group with the control group. In the experimental group, the area of positive staining of collagen type IV and $\alpha$-SMA were markedly increased, as compared with the control group on day 7.

Endothelial cell proliferation in human wounds. The PCI was used for evaluating proliferating endothelial cells, and the results are displayed in Fig. 2A. PCI value was markedly increased between days 1 and 3 in both groups; however, values were significantly elevated in the experimental group compared with the control group at the early stage of wound healing $(\mathrm{P}<0.05$; Fig. 2B). However, the PCI values gradually decreased between days 7 and 15, and values were significantly lower in the experimental group compared with the control group during that time $(\mathrm{P}<0.05)$.

Microvessel density. CD31 and $\alpha$-SMA served as markers for vascular endothelial cells and pericytes, respectively. There was a small quantity of CD31 positive endothelial cells in both groups on day 1 (Fig. 3A). Subsequently, a small quantity of red-stained CD31-positive endothelial cells were detected on day 3 , and the number of endothelial cells was markedly increased on day 7 , and peaked on day 15. Statistical analysis indicated that MVD was significantly higher in the experiment group compared with the control group between days 3 and 15 ( $\mathrm{P}<0.05$; Fig. 3B).

Pericytes count and microvessel pericyte coverage index. Green-stained $\alpha$-SMA positive pericytes were observed, and a small number of pericytes were apparent in both groups on day 3. There were a great number of pericytes discontinuously covering endothelial cells on day 7. Subsequently, on day 15 , the endothelial cells were compactly wrapped with pericytes in the experimental group. By contrast, there were fewer pericytes wrapped with microvessels in the control group (Fig. 3A). Results revealed that the number of pericytes were significantly higher in the experimental group compared with the control group between days 3 and 15 $(\mathrm{P}<0.05$; Fig. 3C). 

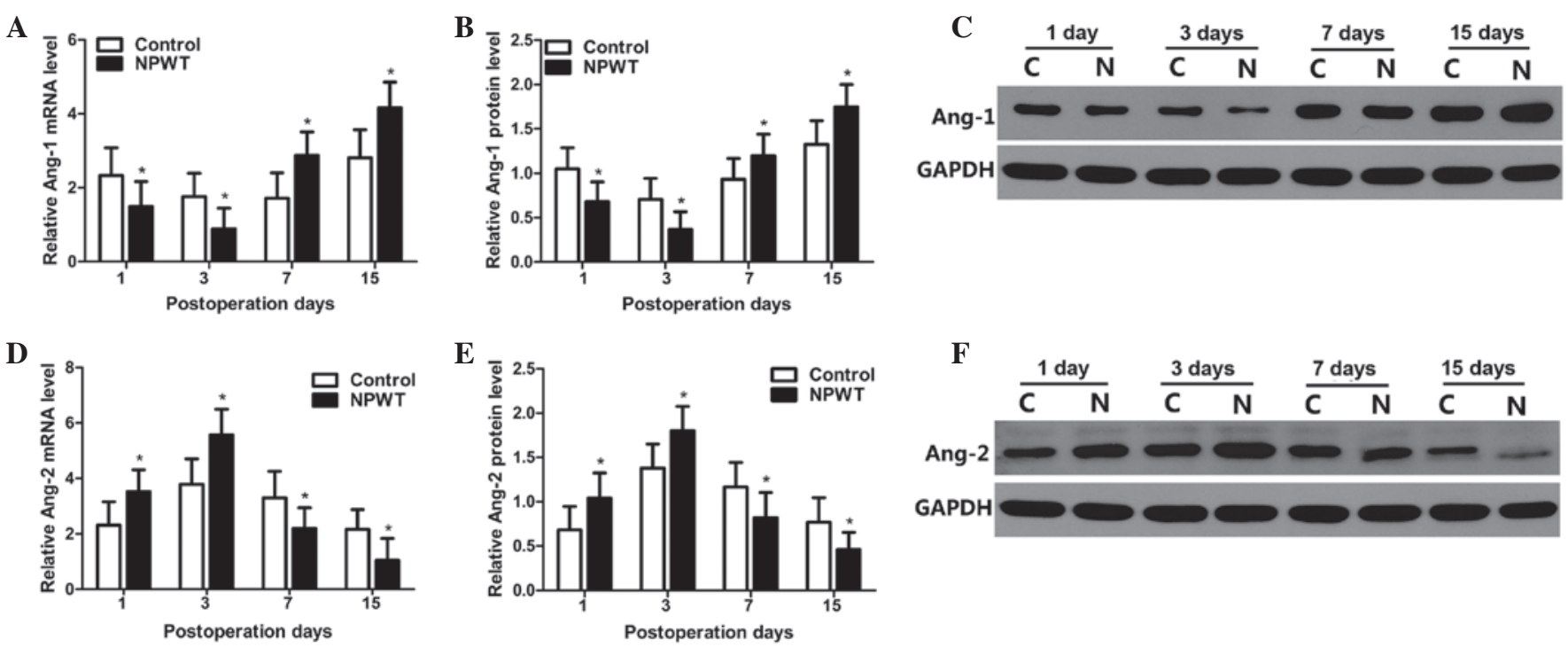

G

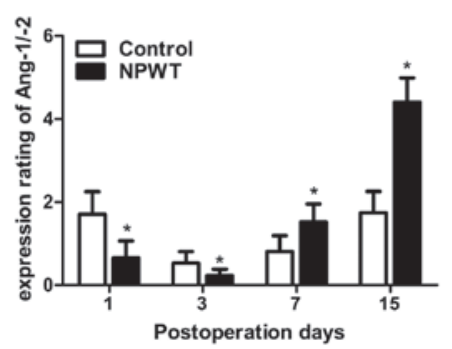

Figure 4. Expression levels of Ang-1 and Ang-2 in the wound during the wound healing process. (A) Ang-1 gene expression levels were assessed by reverse transcription-quantitative polymerase chain reaction (RT-qPCR) in the two groups. (B) Statistical analysis for the protein expression levels of Ang-1 in the two groups. (C) Representative western blots showing the protein levels of Ang-1 in both groups. (D) RT-qPCR analysis gene levels of Ang-2 in the two groups. (E) Statistical analysis protein levels of Ang-2 in the two groups. (F) Representative western blots showing protein expression levels of Ang-2 in the two groups. (G) Protein expression ratio of Ang-1/Ang-2. All statistical analysis results are expressed as the mean \pm standard deviation. "P $<0.05$ vs. control. Ang, angiogenin; NPWT, negative pressure wound therapy.

MPI was used to assess maturity of new blood vessels and results showed there was no statistically significant difference on day 1 between the groups. Subsequently, MPI was significantly increased in both groups between days 7 and 15 , in contrast to the experimental group, where the MPI was significantly higher compared control group at the aforementioned time-points $(\mathrm{P}<0.05$; Fig. 3D).

mRNA expression levels of Ang-1, Ang-2 and Ang-1/Ang-2. The RT-qPCR results indicated that mRNA expression levels of Ang-1 gradually decreased between days 1 and 3 in the experimental group, and expression levels were significantly lower compared with the control group at the same time-point $(\mathrm{P}<0.05)$. mRNA expression levels of Ang-1 gradually increased on day 7, and peaked on day 15 in the two groups; however, its expression levels in experimental group were significantly higher compared with the control group between days 7 and 15 ( $\mathrm{P}<0.05$; Fig. 4A). Western blot analysis was used to quantitatively analyze protein expression levels of Ang-1, as indicted in Fig. 4B. Results revealed that protein expression level trends of Ang-1 were in agreement with the mRNA expression profile in both groups. Relevant results regarding statistical analysis are displayed in Fig. 4C.

The mRNA expression levels of Ang-2 are shown in Fig. 4D. Results indicated that mRNA expression levels of Ang-2 gradually increased in the experimental group from the 1st to the 3rd day, where levels peaked. Furthermore, levels were significantly higher compared with the control group during the aforementioned time-point $(\mathrm{P}<0.05)$. The mRNA expression levels of Ang-2 sharply decreased between days 7 and 15, with levels in the experimental group significantly lower compared with the control group $(\mathrm{P}<0.05)$. Protein expression level trends for Ang-2 were consistent with its mRNA expression level profile in the two groups. Results of statistical analysis performed are displayed in Fig. 4E and F.

The expression ratio of Ang-1/Ang-2 is displayed in Fig. 4G. Results indicated that the expression ratio was significantly lower in the experimental group compared with the control group days 1 and $3(\mathrm{P}<0.05)$; however, this ratio was gradually increased from between days 7 and 15, and maintained a higher expression level, whilst the expression ratio of Ang-1/Ang-2 in the experimental group was significantly higher compared with the control group $(\mathrm{P}<0.05)$.

Expression levels of pTie-2, $\alpha$-SMA and collagen type IV. The tyrosine phosphorylated protein expression levels of Tie-2 were detected by western blot analysis. The changes in protein expression levels pTie-2 are displayed in Fig. 5A, and the results indicate that the expression levels of pTie-2 gradually decreased between days 1 and 3 in both groups, whilst expression levels in the experimental group were significantly lower compared with the control group $(\mathrm{P}<0.05$; Fig. 5B). Subsequently, 

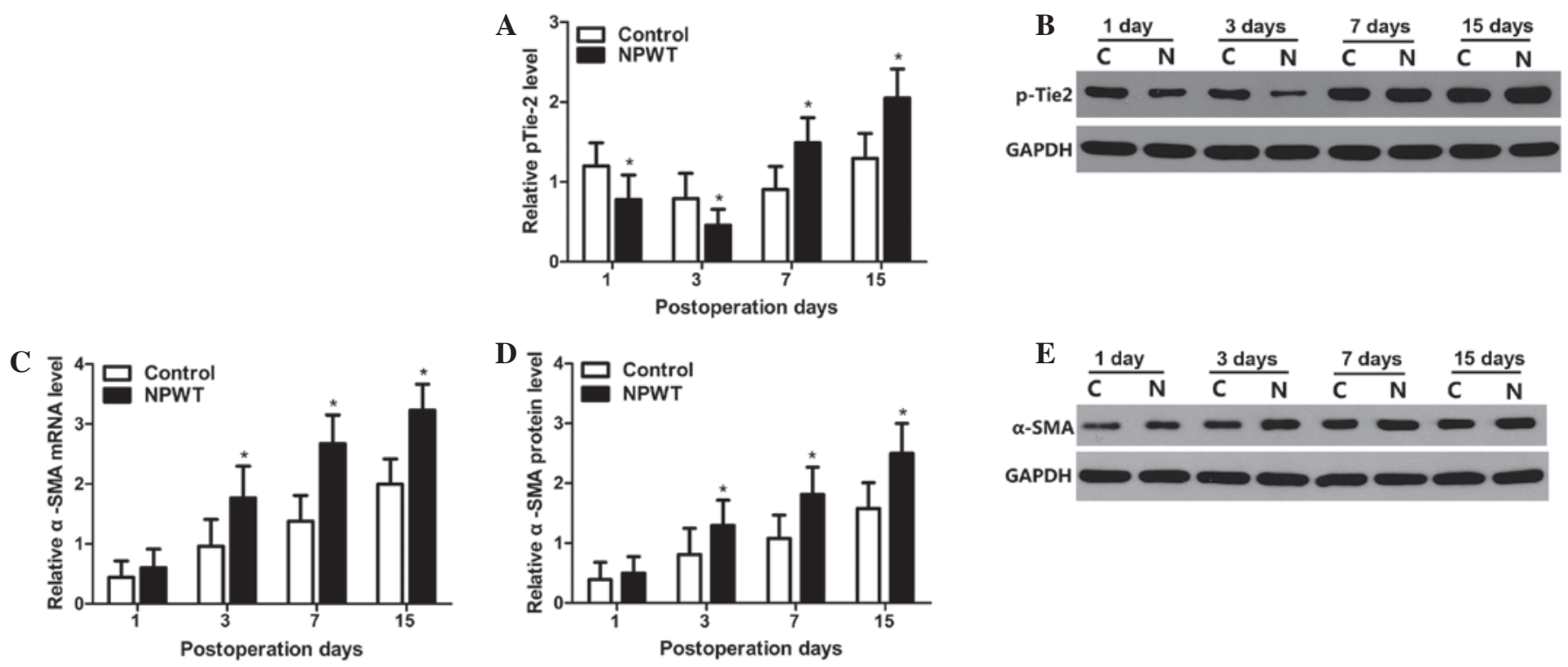

F

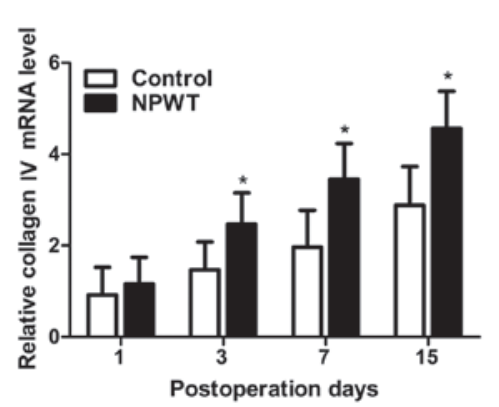

G

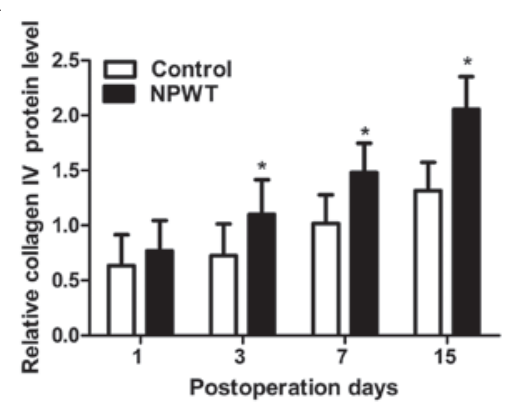

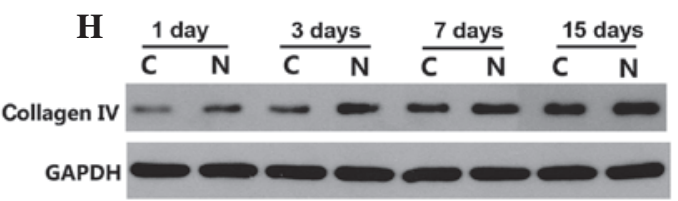

Figure 5. Expression levels of pTie-2 and $\alpha$-SMA in both groups during wound healing. (A) pTie-2 protein expression levels were quantified ("P $<0.05$ ). (B) Representative western blots showing the protein levels of pTie-2 in both groups. (C) Reverse transcription-quantitative polymerase chain reaction (RT-qPCR) was used to assess the expression levels of $\alpha$-SMA mRNA. (D) Quantitative analysis of protein expression levels of $\alpha$-SMA in both groups $\left({ }^{*} \mathrm{P}<0.05\right)$. (E) Representative western blots showing the protein levels of $\alpha$-SMA in both groups. (F) Collagen type IV mRNA expression level was assessed using RT-qPCR. (G) Collagen type IV protein expression levels were quantified in different groups ( $\left.{ }^{*} \mathrm{P}<0.05\right)$. $(\mathrm{H})$ Representative western blots showing the protein levels of collagen type IV in the two groups ("P<0.05). $\alpha$-SMA, $\alpha$-smooth muscle actin; NPWT, negative pressure wound therapy.

expression levels gradually increased between days 7 and 15 , and the expression levels of pTie-2 in the experimental group was significantly higher compared with the control group $(\mathrm{P}<0.05)$. The mRNA and protein expression level changes in $\alpha$-SMA are displayed in Fig. 5C-E. The results indicated that $\alpha$-SMA expression levels gradually increased between days 3 and 15 , and expression levels were significantly higher in the experimental group compared with the control group $(\mathrm{P}<0.05)$. The mRNA and protein expression level changes of collagen type IV are displayed in Fig. 5F-H. The expression levels of collagen type IV presented trends that were concordant with the $\alpha$-SMA expression profile in both groups.

\section{Discussion}

NPWT has been routinely used for the treatment of various chronic and acute wounds, bone exposure, pressure ulcers and diabetes mellitus wounds. Previous studies have demonstrated that NPWT is able to promote the formation of granulation tissue and increase the amount of angiogenesis (30-32), accelerate wound neovascularization, increase blood flow perfusion and thus accelerate wound re-epithelialization $(33,34)$, in addition to accelerating the speed of wound healing in wounds in rats $(34,35)$. However, the investigation of new blood vessel maturation and the relevant signal pathway subsequent to NPWT in human wound, is infrequent and limited. In the present study, the changes in microvasculature were investigated at various stages following NPWT in human wounds, and whether NPWT was capable of promoting the maturation of new blood vessels was explored, in addition to the corresponding signal pathway. Furthermore, the relevant association between the maturation of nascent blood vessels and the prognosis of wounds were investigated and analyzed.

In normal and mature blood vessels, basement membrane is shared by the vascular endothelial tube and pericytes $(11,36)$. Mature microvessels, predominantly characterized by the vascular endothelial tube, were covered with abundant pericytes and the basement membrane $(11,20,37)$. Pericytes serve as a specific structural component and marker of vessel maturation, wrapping around microvascular endothelial cells $(11,38)$. Furthermore, pericytes are able to contact endothelial cells through the hole of the basement membrane and form special cell-cell contact, termed peg-and-socket contact (39). Pericytes and endothelial cells communicate with each other by the peg-and-socket contacts, and control the proliferation and differentiation of endothelial cells $(37,39)$, 
and transmit mechanical contractile forces to affect blood flow perfusion $(20,40)$. The characterization of pericytes was previously problematic due to the absence of a specific molecular marker; however, several recent studies have indicated that $\alpha$-SMA is able to serve as marker of pericytes $(20,37,39,41)$. Consequently, $\alpha$-SMA served as a pericyte marker in the present study.

To further elucidate the method by which NPWT is able to regulate the process of angiogenesis and vessel maturation in human wounds, the corresponding expression levels of pro-angiogenesis factor Ang-2, and pro-maturation factor Ang-1, which are able to regulate the sprouting of new blood vessels and their maturation, were detected (42). Previous studies have demonstrated that the process of angiogenesis and vessel maturation is regulated by the Ang/Tie-2 system in the wounds of rats $(11,39,41)$. It has been demonstrated that Ang-1 functions as a pericyte-derived blood vessels stabilizer and pro-maturation factor; binding to the Tie- 2 receptor and serving an important role in sustaining quiescent microvasculature (43). In the present study, RT-qPCR and western blot analysis indicated that expression levels of Ang-1 and pTie-2 were gradually reduced in the early stage of wound healing following NPWT. However, in the control group the expression levels of Ang-1 and pTie-2 were significantly higher compared with the experimental group in the early stage. By contrast, as an antagonist, Ang-2 inhibited the phosphorylation of Tie-2 in endothelial cells induced by Ang-1 $(3,16,19)$, and induced the destabilization and regression of microvasculature (44). Brudno et al (42) proposed that a destabilized wound microenvironment would be able to facilitate vessel sprouting and angiogenesis. The results in the present study indicated that, in the experimental group, the expression levels of Ang-2 were significantly higher compared with the control group in the early stage of wound healing following NPWT. Furthermore, the present study observed that the lower expression ratio of Ang-1/Ang-2, and the MVD and PCI were significantly higher in the experimental group compared with the control group in the early stage. The aforementioned results suggest that microvessels were regressive and destabilized in the early stage of wound healing. The present data demonstrated that NPWT was able to preferentially promote microvessel regression and destabilization at the early stage, and thus promote vascular endothelial cell sprouting and proliferation, and increase the amount of angiogenesis.

Subsequent to microvascular endothelial lumen formation, stabilization and maturation of the nascent blood vessels takes place in the later stage of wound healing. Pro-maturation factor Ang-1 has an important role in promoting recruitment of mural cell and blood vessel maturation in the later stage of wound healing (45). Previous studies have demonstrated that pericytes are an important component of microvessel maturation, and support microvascular structural integrity and functional stabilization $(46,47)$. Blood vessel maturation is predominately characterized by an abundance of pericytes wrapping around vascular endothelial tubes $(3,11)$. Previous studies have demonstrated that immature vessels induced vessel hemorrhage, tissue oedema and vessels occlusion, and eventually led to obstruction of the transportation of nutrients and oxygen $(18,48)$. The results of the present study indicated that at the later stage of wound healing, the expression levels of Ang-1 and pTie-2 gradually increased in the experimental group following NPWT, and the difference was statistically significant compared with the control group. However, expression levels of Ang-2 gradually decreased in the experimental group, compared with the control group in the later stage of the wound healing process. Furthermore, the results for the expression ratio of Ang-1/Ang-2 were significantly increased in the NPWT group, as compared with the control group at a later stage of wound healing. Additionally, in the experimental group, the blood flow perfusion was significantly increased and $\alpha$-SMA and collagen IV also increased gradually, thus MPI was relatively higher in the experimental group between days 7 and 15 . Results suggested that microvessels were gradually stabilized in the later stage, and the stabilized microvascular microenvironment contributed to mediate the recruitment of pericytes to vessel tubes, and promoted the maturation of new blood vessels. NPWT predominately promoted microvessel stabilization and maturation in the later stage of wound healing in human wounds, and thus increased blood flow perfusion and accelerated the speed of wound healing.

Finally, all wounds were covered with skin grafting or underwent the transposition flap technique according to the quality of granulation tissue following NPWT or petrolatum gauze treatment. Previous research has demonstrated that a granulation tissue wound may be treated via covering with skin grafting, which is preferable to the transposition flap when red, fresh and abundant tissue granulation is detected $(22,49)$. Combining the results of patient demographics, laboratory results of patient cohorts and blood flow changes, the present study indicated that in the experimental group, there was a greater number of cases of skin grafting and blood flow perfusion of the wound, and a lower number of cases of requiring the transposition flap technique, in addition to a shorter hospital stay, compared with the control group. The aforementioned results may be associated with blood vessel maturation. Due to the gradual maturation of microvessels at the later stage of wound healing in the experimental group, in addition to the increased number of pericytes, which serve as a blood flow regulators, in later stage of wound healing $(22,50)$, blood flow perfusion was increased at the site of the wound. As a result, notable granulation tissue was generated, and subsequently the wound healing process was markedly accelerated by NPWT. It was further suggested that NPWT was able to promote angiogenesis and microvessel maturation at different stages during wound healing, and increased blood flow perfusion, which may eventually be able to influence wound prognosis.

In summary, NPWT was able to promote microvessel destabilization and regression, and consequently promote vessel sprouting and increase the quantity of microvessels in the early stage of wound healing. In addition, NPWT was able to promote the structural integrity and functional stabilization of microvessels, and then promote microvascular maturation during the later stage of human wound healing. The present study also demonstrated that mature microvessels were able to influence wound prognosis in human wounds.

\section{Acknowledgements}

The present study was supported by the National Natural Science Foundation of China (grant no. 81572163) and by Hubei 
National Natural Science Fund projects (grant no. 2014CFB751). The authors would also like to acknowledge the Wuhan VSD Medical Science \& Technology, Co., Ltd. (Wuhan, China) for supplying the vacuum material. Finally, thanks is given to the Medical Science Experimentation Center of Wuhan University for providing the experimental equipment.

\section{References}

1. Zhang DM, Yang ZH, Zhuang PL, Wang YY, Chen WL and Zhang B: Role of negative-pressure wound therapy in the management of submandibular fistula after reconstruction for osteoradionecrosis. J Oral Maxillofac Surg 74: 401-405, 2016.

2. Ram M, Singh V, Kumawat S, Kumar D, Lingaraju MC, Uttam Singh T, Rahal A, Kumar Tandan S and Kumar D: Deferoxamine modulates cytokines and growth factors to accelerate cutaneous wound healing in diabetic rats. Eur J Pharmacol 764: 9-21, 2015.

3. Qin D, Trenkwalder T, Lee S, Chillo O, Deindl E, Kupatt C and Hinkel R: Early vessel destabilization mediated by Angiopoietin-2 and subsequent vessel maturation via Angiopoietin-1 induce functional neovasculature after ischemia. PLoS One 8: e61831, 2013.

4. Hinkel R, Trenkwalder T and Kupatt C: Gene therapy for ischemic heart disease. Expert Opin Biol Ther 11: 723-737, 2011

5. Jain RK: Molecular regulation of vessel maturation. Nat Med 9: 685-693, 2003.

6. Greenberg JI, Shields DJ, Barillas SG, Acevedo LM, Murphy E, Huang J, Scheppke L, Stockmann C, Johnson RS, Angle N and Cheresh DA: A role for VEGF as a negative regulator of pericyte function and vessel maturation. Nature 456: 809-813, 2008.

7. Kupatt C, Hinkel R, Pfosser A, El-Aouni C, Wuchrer A, Fritz A, Globisch F, Thormann M, Horstkotte J, Lebherz C, et al: Cotransfection of vascular endothelial growth factor-A and platelet-derived growth factor-B via recombinant adeno-associated virus resolves chronic ischemic malperfusion role of vessel maturation. J Am Coll Cardiol 56: 414-422, 2010.

8. Kilarski WW, Samolov B, Petersson L, Kvanta A and Gerwins P: Biomechanical regulation of blood vessel growth during tissue vascularization. Nat Med 15: 657-664, 2009.

9. Patel-Hett S and D'Amore PA: Signal transduction in vasculogenesis and developmental angiogenesis. Int J Dev Biol 55: 353-363, 2011.

10. Dor Y, Djonov V, Abramovitch R, Itin A, Fishman GI, Carmeliet P, Goelman G and Keshet E: Conditional switching of VEGF provides new insights into adult neovascularization and pro-angiogenic therapy. Embo J 21: 1939-1947, 2002.

11. Zhao J, Chen L, Shu B, Tang J, Zhang L, Xie J, Qi S and Xu Y: Granulocyte/macrophage colony-stimulating factor influences angiogenesis by regulating the coordinated expression of VEGF and the Ang/Tie system. PLoS One 9: e92691, 2014.

12. Greene AK, Puder M, Roy R, Arsenault D, Kwei S, Moses MA and Orgill DP: Microdeformational wound therapy: Effects on angiogenesis and matrix metalloproteinases in chronic wounds of 3 debilitated patients. Ann Plast Surg 56: 418-422, 2006.

13. Chen SZ, Li J, Li XY and Xu LS: Effects of vacuum-assisted closure on wound microcirculation: An experimental study. Asian J Surg 28: 211-217, 2005.

14. Grimm A, Dimmler A, Stange S, Labanaris A, Sauer R, Grabenbauer G and Horch RE: Expression of HIF-1 alpha in irradiated tissue is altered by topical negative-pressure therapy. Strahlenther Onkol 183: 144-149, 2007.

15. Labler L, Rancan M, Mica L, Harter L, Mihic-Probst D and Keel M: Vacuum-assisted closure therapy increases local interleukin-8 and vascular endothelial growth factor levels in traumatic wounds. J Trauma 66: 749-757, 2009.

16. Reiss Y, Droste J, Heil M, Tribulova S, Schmidt MH, Schaper W, Dumont DJ and Plate KH: Angiopoietin-2 impairs revascularization after limb ischemia. Circ Res 101: 88-96, 2007.

17. Gaengel K, Genove G, Armulik A and Betsholtz C: Endothelial-mural cell signaling in vascular development and angiogenesis. Arterioscler Thromb Vasc Biol 29: 630-638, 2009.

18. Fagiani E and Christofori G: Angiopoietins in angiogenesis. Cancer Lett 328: 18-26, 2013.

19. Maisonpierre PC, Suri C, Jones PF, Bartunkova S, Wiegand SJ, Radziejewski C, Compton D, McClain J, Aldrich TH, Papadopoulos N, et al: Angiopoietin-2, a natural antagonist for Tie2 that disrupts in vivo angiogenesis. Science 277: 55-60, 1997.
20. Armulik A, Genové G and Betsholtz C: Pericytes: Developmental, physiological and pathological perspectives, problems and promises. Dev Cell 21: 193-215, 2011.

21. Lee HJ, Cho CH, Hwang SJ, Choi HH, Kim KT, Ahn SY, Kim JH, Oh JL, Lee GM and Koh GY: Biological characterization of angiopoietin-3 and angiopoietin-4. Faseb J 18: 1200-1208, 2004.

22. Zhou M, Qi B, Yu A, Pan Z, Zhu S, Deng K and Tao S: Vacuum assisted closure therapy for treatment of complex wounds in replanted extremities. Microsurgery 33: 620-624, 2013.

23. Matsunaga T, Warltier DC, Tessmer J, Weihrauch D, Simons M and Chilian WM: Expression of VEGF and angiopoietins-1 and -2 during ischemia-induced coronary angiogenesis. Am J Physiol Heart Circ Physiol 285: H352-H358, 2003.

24. Weidner N: Tumoural vascularity as a prognostic factor in cancer patients: The evidence continues to grow. J Pathol 184: 119-122, 1998.

25. Yonenaga Y, Mori A, Onodera H, Yasuda S, Oe H, Fujimoto A, Tachibana T and Imamura M: Absence of smooth muscle actin-positive pericyte coverage of tumor vessels correlates with hematogenous metastasis and prognosis of colorectal cancer patients. Oncology 69: 159-166, 2005.

26. Zhao W, Jiang AH, Li CY, Yang WZ, Xu CC and Liu ZG: Pericytes are correlated with the permeability of rat corneal neovascular vessels induced by alkali burn. Chin Med J (Engl) 120: 274-279, 2007.

27. O'Keeffe MB, Devlin AH, Burns AJ, Gardiner TA, Logan ID, Hirst DG and McKeown SR: Investigation of pericytes, hypoxia and vascularity in bladder tumors: Association with clinical outcomes. Oncol Res 17: 93-101, 2008.

28. Kalinski T, Sel S, Kouznetsova I, Röpke M and Roessner A: Heterogeneity of angiogenesis and blood vessel maturation in cartilage tumors. Pathol Res Pract 205: 339-345, 2009.

29. Livak KJ and Schmittgen TD: Analysis of relative gene expression data using real-time quantitative PCR and the 2(-Delta Delta C(T)) method. Methods 25: 402-408, 2001.

30. Armstrong DG and Lavery LA; Diabetic Foot Study Consortium: Negative pressure wound therapy after partial diabetic foot amputation: A multicentre, randomised controlled trial. Lancet 366: 1704-1710, 2005

31. Apelqvist J,Armstrong DG, Lavery LA and Boulton AJ: Resource utilization and economic costs of care based on a randomized trial of vacuum-assisted closure therapy in the treatment of diabetic foot wounds. Am J Surg 195: 782-788, 2008.

32. Blume PA, Walters J, Payne W, Ayala J and Lantis J: Comparison of negative pressure wound therapy using vacuum-assisted closure with advanced moist wound therapy in the treatment of diabetic foot ulcers: A multicenter randomized controlled trial. Diabetes Care 31: 631-636, 2008

33. Tuncel U, Turan A, Markoc F, Erkorkmaz U, Elmas C and Kostakoglu N: Loofah sponge as an interface dressing material in negative pressure wound therapy: Results of an in vivo study. Ostomy Wound Manage 60: 37-45, 2014.

34. Xia CY, Yu AX, Qi B, Zhou M, Li ZH and Wang WY: Analysis of blood flow and local expression of angiogenesis-associated growth factors in infected wounds treated with negative pressure wound therapy. Mol Med Rep 9: 1749-1754, 2014.

35. Li X, Liu J, Liu Y, Hu X, Dong M, Wang H and Hu D: Negative pressure wound therapy accelerates rats diabetic wound by promoting agenesis. Int J Clin Exp Med 8: 3506-3513, 2015.

36. Kim N and Cho SG: Clinical applications of mesenchymal stem cells. Korean J Intern Med 28: 387-402, 2013.

37. Gokcinar-Yagci B, Uçkan-Çetinkaya D and Çelebi-Saltik B: Pericytes: Properties, functions and applications in tissue engineering. Stem Cell Rev 11: 549-559, 2015.

38. Stratman AN, Malotte KM, Mahan RD, Davis MJ and Davis GE: Pericyte recruitment during vasculogenic tube assembly stimulates endothelial basement membrane matrix formation. Blood 114: 5091-5101, 2009.

39. Ribatti D, Nico B and Crivellato E: The role of pericytes in angiogenesis. Int J Dev Biol 55: 261-268, 2011.

40. Bergers G and Song S: The role of pericytes in blood-vessel formation and maintenance. Neuro Oncol 7: 452-464, 2005.

41. Aguilera KY and Brekken RA: Recruitment and retention: Factors that affect pericyte migration. Cell Mol Life Sci 71: 299-309, 2014.

42. Brudno Y, Ennett-Shepard AB, Chen RR, Aizenberg M and Mooney DJ: Enhancing microvascular formation and vessel maturation through temporal control over multiple pro-angiogenic and pro-maturation factors. Biomaterials 34: 9201-9209, 2013.

43. Thomas M and Augustin HG: The role of the Angiopoietins in vascular morphogenesis. Angiogenesis 12: 125-137, 2009. 
44. Scharpfenecker M, Fiedler U, Reiss Y and Augustin HG: The Tie-2 ligand angiopoietin-2 destabilizes quiescent endothelium through an internal autocrine loop mechanism. J Cell Sci 118: 771-780, 2005.

45. Brindle NP, Saharinen P and Alitalo K: Signaling and functions of angiopoietin-1 in vascular protection. Circ Res 98: 1014-1023, 2006

46. Fagiani E, Lorentz P, Kopfstein L and Christofori G: Angiopoietin-1 and -2 exert antagonistic functions in tumor angiogenesis, yet both induce lymphangiogenesis. Cancer Res 71: 5717-5727, 2011.

47. Hirschi KK, Rohovsky SA, Beck LH, Smith SR and D'Amore PA: Endothelial cells modulate the proliferation of mural cell precursors via platelet-derived growth factor-BB and heterotypic cell contact. Circ Res 84: 298-305, 1999.
48. Bhushan M, Young HS, Brenchley PE and Griffiths CE: Recent advances in cutaneous angiogenesis. Br J Dermatol 147: 418-425, 2002

49. Stannard JP, Singanamala N and Volgas DA: Fix and flap in the era of vacuum suction devices: What do we know in terms of evidence based medicine? Injury 41: 780-786, 2010.

50. Hamilton NB, Attwell D and Hall CN: Pericyte-mediated regulation of capillary diameter: A component of neurovascular coupling in health and disease. Front Neuroenergetics 2: 5, 2010 . 